\title{
L'Âge du Bronze dans les pays de la Loire moyenne
}

Jean Gasco et Michel Dauvois

\section{OpenEdition}

\section{Journals}

Édition électronique

URL : http://journals.openedition.org/rao/1427

DOI : $10.4000 /$ rao. 1427

ISSN : 1775-3732

\section{Éditeur}

Presses universitaires de Rennes

\section{Édition imprimée}

Date de publication : 31 décembre 2010

Pagination : 224-229

ISBN : 978-2-7535-1383-9

ISSN : 0767-709X

\section{Référence électronique}

Jean Gasco et Michel Dauvois, «L'Âge du Bronze dans les pays de la Loire moyenne », Revue archéologique de l'Ouest [En ligne], 27 | 2010, mis en ligne le 25 février 2012, consulté le 03 décembre 2020. URL : http://journals.openedition.org/rao/1427 ; DOI : https://doi.org/10.4000/rao.1427

Ce document a été généré automatiquement le 3 décembre 2020

Tous droits réservés 


\title{
L'Âge du Bronze dans les pays de la Loire moyenne
}

\author{
Jean Gasco et Michel Dauvois
}

\section{RÉFÉRENCE}

CORDIER, G., 2009 - L'Âge du Bronze dans les pays de la Loire moyenne. Joué-les-Tours, La Simarre (édition/diffusion à compte d'auteur : G. Cordier, Sublaines, 37310 Reignac sur Indre), 702 p., XXII pl. h-t. (ISBN 2-902559-72-0 ; $125 €$ )

\section{NOTE DE L'ÉDITEUR}

Deux collègues ayant indépendamment souhaité présenter cet important volume, nous leur ouvrons simultanément ces colonnes, d'autant plus volontiers que leurs regards s'avèrent largement complémentaires.

1 L'ouvrage de G. Cordier, retraité (le mot est plaisant) tourangeau, est une somme impressionnante avec ses 702 pages, 22 planches et 459 figures, lesquelles représentent des milliers d'heures de dessins et de mises au net affûtées, précises et, on le sait, très artistiques. Le lecteur feuilletant l'ouvrage en tirera ainsi d'abord un grand plaisir esthétique.

L'auteur, membre actif de la Société préhistorique française depuis 1942 et entré au CNRS en 1966 pour étudier l'Âge du Bronze de sa région, signe ici un impressionnant ouvrage d'inventaire et d'analyse en réunissant les études - parfois synthétisées - de très nombreux sites et documents ${ }^{1}$. C'est l'aboutissement d'un ancien projet engagé par la révision des dépôts et trouvailles de l'Âge du Bronze connus en Touraine dans les années 1960, projet maintenu durant près d'un demi-siècle malgré la prolifération des données nouvelles. La multiplication des découvertes et l'accroissement des documents (voir la bibliographie arrêtée à 2005 par le jeune homme alors âgé de 81 ans) expliquent 
le considérable bilan d'une cinquantaine d'années de recherche qu'il nous propose aujourd'hui' ${ }^{2}$.

3 Ces données, parfois plus développées, avaient été publiées au coup par coup, et G. Cordier signale pour sa part 66 textes personnels. Parfois éparpillés, anciens ou confidentiels, ces derniers imposaient aux chercheurs qui voulaient les consulter d'innombrables dépouillements et souvent, pour les archéologues extra-régionaux, des tractations entre collègues mieux informés. L'auteur, qui savait par cette publication rendre ainsi service à sa communauté, a souhaité, sans aucune aide, matérialiser à sa seule convenance le travail d'une partie de sa vie. En donnant la seule priorité aux faits et aux objets, avec méthode. L'entreprise est nous l'avons dit impressionnante. Il réunit ici en effet de nombreuses études engagées dès les années 1950, avec l'impulsion et l'amitié de J.-P. Millotte, de R. Riquet et de M. Gruet, élargies depuis la Touraine à l'ensemble du Bassin moyen de la Loire.

4 Le savant est un homme de Touraine, homme de terrain et d'analyse ${ }^{3}$. Fin prospecteur, il a consacré sa vie à cette région avec un inventaire des mégalithes d'Indre-et-Loire (1963, révisé en 1984) puis celui des instruments perforés $(1957,1970,1984)$. Il accomplit aussi les fouilles des tombes de Chissay-en-Touraine (1961-1967), Sublaines (1964-1968, 1975) et l'étude de nombreux autres sites. Expert auprès de nombreux musées ou sociétés savantes, on lui doit en particulier les publications de très nombreux dépôts d'objets de bronze dont les dessins évoquent la précision graphique des graveurs du XIX siècle : Azay-le-Rideau en 1959, les Bourgeons rouges en 1966, la Fontaine de Lucineau en 1978, Sublaines en 1988, le Petit-Vilatte en 1996, le Gué-deLongroi en 1984 et celle de quantité d'objets tirés de la Loire ou de ses rives (une cinquantaine rien qu'entre 1967 et 1972). Ces documents sont présents dans le volume.

5 Il est donc présomptueux d'accepter de rédiger un compte rendu d'une telle oeuvre, sinon à rappeler les modalités de son élaboration et à commenter, comme des réflexions personnelles, la présentation de la dynamique de l'Âge du Bronze de cette région. L'ouvrage couvre l'ensemble de la période et traite en fait de la question d'une identité régionale, celle de l'autonomie des évolutions des populations dans le cadre d'échanges et d'impacts culturels. Les sources documentaires disponibles sont essentiellement métalliques et conduisent donc à n'observer que les comportements d'une élite sociale produisant ou consommant des objets de bronze, mobiliers très inégalement répartis, il faut le souligner, dans la société agricole du IIe millénaire.

6 La région du Bassin de la Loire est un espace ouvert avec, comme seul " compartimentage» actuellement décelable, des cadres provinciaux historiques encore très prégnants et le lien fort d'un grand fleuve européen et de ses affluents. "L'uniformité de ces pays de plaine» en fait une région avant tout "réceptrice». Durant le $\mathrm{II}^{\mathrm{e}}$ millénaire, comme dans de nombreux autres territoires de la France, l'indéniable force culturelle du centre est-européen (Allemagne du Sud, Suisse, France orientale) y a précocement imposé sa marque et, à la fin de l'Âge du Bronze, cette région est souvent considérée, malgré une forte identité "atlantique", comme une zone éloignée de sujétion. L'histoire même de la discipline protohistorique orientaloseptentrionale (écoles archéologiques, sériations céramiques continentales, bases précoces de l'établissement des chronologies) a probablement amplifié la perception que l'on a de cette réalité. Car la région de la Loire est aussi partie prenante de l'orbe occidentale et, en particulier, la puissance des sociétés métalliques de cette aire y est largement perceptible. C'est sans doute cet angle d'analyse qui valorise et met en avant 
l'originalité ligérienne quand la pratique bronzière y est pleinement établie. La linéarité de son évolution historique au cours du $\mathrm{II}^{\mathrm{e}}$ millénaire, comme une région du finistère européen de l'Âge du Bronze, ne reste toutefois appréciable qu'à certaines échelles, quand d'autres peuvent éclairer des cheminements locaux plus complexes.

7 Un formidable biais existe cependant qui ne permet pas d'évaluer l'ensemble de la société régionale de l'Âge du Bronze et favorise alors une sourde idée de dépendance et de périphérie. Malgré un timide renouveau des recherches et quelques notables exceptions rapidement présentées (comme les fouilles inédites de Méhers en Loir-etCher pour le Bronze ancien, celles du Camp-Allaric d'Aslonnes dans la Vienne ${ }^{4}$, de l'Assignat de Barrou en Indre-et-Loire ou du Coteau-de-Montigné à Coulon dans les Deux-Sèvres pour le Bronze final), ce ne sont pas les habitats qui illustrent l'Âge du Bronze du Bassin de la Loire. Et les découvertes récentes, importantes et inédites, sont peu nombreuses 5 . Ce ne sont pas plus les sites funéraires anciens dont les inventaires de G. Cordier indiquent le petit nombre (deux tombes pour le Bronze ancien, trois pour le Bronze moyen). Seuls les sites de tombes plates, la plupart étudiés au xixe siècle, deux tumulus célèbres (Sublaines) et surtout 17 nécropoles à incinération ne réunissant toutefois que 106 tombes, permettent de se familiariser par exemple avec les rites sépulcraux ou la céramique de la fin de l'Âge du Bronze ligérien ${ }^{6}$. Ce sont donc les découvertes isolées et les dépôts d'objets de bronze qui éclairent à l'heure actuelle, avec toutes les déformations qu'ils instaurent, la société régionale. Ils font l'essentiel de l'étude de G. Cordier.

8 Au cours de l'Âge du Bronze ancien, les vents souffleraient de l'Est, du moins pour la métallurgie. Les lingots arciformes de Bourges, les haches de Villemandeur, les sept hallebardes extraites de la Loire et les productions métalliques ligériennes sont ainsi des objets "occidentaux", d'Allemagne du sud, ou helvétiques. Un lingot-torque décoré à Cléré-du-Bois (Indre) et des lingots-bipennes évoquent aussi des ateliers d'Allemagne centrale ou des régions proches. La céramique de quelques sites confirmerait peut-être, selon G. Cordier d'ailleurs, de telles relations avec les pays rhénans (Amboise, Rochecorbon, Saint-Nicolas-de-Bourgueil) sinon avec le plateau suisse (fig. 37).

9 Mais il ne semble pas que les haches plates aient une telle origine : le centre vendéen ou de basse Loire, où l'on trouve pratiquement les seuls dépôts, en a diffusé progressivement des formes de plus en plus évoluées à l'intérieur des terres, en utilisant le réseau de circulation des vallées fluviales. Tout laisse à penser qu'il existait là des productions locales, comme des indices métallifères dans des régions proches pourraient en faire accepter l'idée (fig. 4).

10 Les spécialistes s'interrogent en fait sur le niveau de dépendance ou d'autonomie de cette métallurgie et analysent son origine. S'agit-il de produits allochtones, mais provenant plutôt d'une tête de pont d'un premier « trafic maritime » de la basse Seine à la Gironde, ou sinon d'échanges soutenus? Des liens sociétaux et culturels avec l'Armorique existent alors à l'évidence, par exemple dans le mode sépulcral en coffre utilisé à Saint-Rimay (Loir-et-Cher). Cette supposée unité atlantique dépasse-t-elle même le cadre régional littoral - en quelque sorte les périmètres du cabotage - si l'on considère aussi la présence dans cette région d'une gargantilla (Saint-Laurs, DeuxSèvres) ou d'autres bijoux en or (diadème de Chalais dans la Vienne), vraisemblablement d'origine ibérique (fig. 30 )? 
11 Cette propension à élargir les aires culturelles ou à tenter d'y apprécier les interactions pousse aussi G. Cordier à considérer une seconde grande force européenne - que l'on qualifie grossièrement de rhodano-méridionale - via le Massif central voire le sud de la France et le Languedoc. Des productions précoces pénètrent alors en effet jusqu'aux confins nord de l'Aquitaine. Cette dernière hypothèse permet d'associer à ce mouvement le poignard méridional de Conie-Molitard (Eure-et-Loir), de s'appuyer sur un poignard chypriote Vendômois (douteux il est vrai), de souligner encore l'existence d'une lame padane de type Remedello (à cinq trous de rivet) égarée dans une tombe à Naveil (Loir-et-Cher). Pour autant quelques objets, aussi emblématiques soient-ils, ne caractérisent pas de supposés liens et a fortiori des dominations culturelles. Et tenter d'évaluer l'origine et surtout le poids d'un éventuel commerce, d'échanges, d'emprunts ou d'influences entre des régions lointaines à partir de séries limitées est un effort louable mais sans doute une gageure.

12 Constatons que la vallée de la Loire est au début de l'Âge du Bronze pleinement européenne, irriguée de multiples apports, y compris maritimes, y compris à très longue distance. Cette ouverture, probablement aussi en réminiscence d'une certaine mobilité antérieure, tient peut-être aux caractéristiques régionales et à la présence de la Loire et de ses vallées affluentes pénétrables, milieux très favorables lorsque les échanges de biens s'intensifient. Elle est surtout à attribuer à l'invention métallurgique elle-même, qui vient brutalement de transformer les rapports sociaux et territoriaux entre populations. Avec la conjonction des deux phénomènes, les ateliers et les commerçants sous l'emprise des innovations et de la concurrence ont d'ailleurs très rapidement complexifié la diffusion des objets recherchés ${ }^{7}$. Les échanges à longue distance, largement banalisés désormais, ne minimisent pas cependant les atouts régionaux et les capacités des producteurs locaux à prendre les informations, à en digérer ou à en produire tous les assortiments.

Il semble qu'on en ait en tous cas la preuve au cours du Bronze moyen. La société a alors totalement intégré le rapport nouveau au métal des peuples européens, tant dans sa fabrication locale, son échange, son acquisition ou son utilisation quotidienne que dans sa thésaurisation, temporaire ou non (dépôts de Luynes, des environs de Tours (Evans-Franks), des Montils, de Saint-Gervais-la-Forêt, de Férolles, de Tigy, de Lion-enSullias, de Saint-Martin-sur-Ocre). La métallurgie est non seulement généralisée dans le territoire étudié (fig. 146, 454), comme par exemple une quarantaine de haches à rebords et près de 150 haches à talon l'indiquent, mais elle acquiert alors une forte identité avec la pratique de la conservation en dépôts. L'analyse proposée par G. Cordier montre alors la totale intégration macro-régionale « atlantique » de l'ensemble du bassin de la Loire. Et ceci vaut au-delà de la production métallurgique puisque la céramique le confirme souvent (influences armoricaines au Thoureil, à Trèves-Cunault, à Fondettes, etc.). Ces "influences" des régions proches, qui prouvent en fait des communautés de modes culturelles appliquées à tel ou tel objet, sont largement assimilées, souvent selon la règle de la proximité : par exemple, les haches à forts rebords sont plutôt vendéennes, les haches à talon longilignes de mode bretonne. La métallurgie normande impacte les productions du nord de la Loire - dépôts de Longnyau-Perche (Orne) et du Gué-de-Longroi (Eure-et-Loir) - quant celle du groupe de Tréboul - Saint-Brandan est encore plus diffuse.

14 L'activité métallurgique locale reste pourtant méconnue. Mais les ressources métallifères potentielles du plateau central (Limousin, Creuse) auraient pu alimenter 
les ateliers régionaux (moules et creusets de Gartempe et Sainte-Feyre). Les productions locales (moules de Martizay et de Saint-Denis-les-Ponts) et la pratique des dépôts de type Centre-Ouest (Pamproux) traduisent petitement la place importante des ateliers métalliques dans la société.

Dans le bassin de la Loire, la fin de l'Âge du Bronze moyen est en tous cas devenue pour certains une période faste, comme le dépôt de Chéry, en Berry, ou le dépôt angevin des Ponts-de-Cé en apportent la preuve. Des individus ou des groupes, dont on ignore tout de l'origine de leur prospérité, ne peuvent se contenter d'objets régionaux trop communs ; ils deviennent plus que jamais des clients potentiels aux échanges lointains. Et ce sont quelques objets exceptionnels qui attestent de la fortune de cette petite élite régionale : hache «bohémienne » de Châteauneuf-sur-Loire, hache de parade d'Azay-leRideau (rapprochée de la Bavière ou du Rhin moyen - photographie de couverture de l'ouvrage) et cette « merveille d'orfèvrerie et objet de haute valeur cultuelle impliquant des relations lointaines vers l'Est » qu'est le « cône d'or » d'Avanton" ${ }^{8}$.

L'appétence métallurgique irradie donc cette région et ce sont des productions variées voire éclectiques qui vont désormais alimenter de très nombreux dépôts régionaux tout au long de la fin de l'Âge du Bronze. Cette pratique d'enfouissements est considérée comme un des traits culturels distinctifs du Bronze final « atlantique ». Leur fréquence et leurs volumes sont désormais importants. Ainsi, en Touraine, se multiplient des dépôts massifs d'objets de bronze: au premier rang, viennent celui d'Amboise, attribuable à la transition Bronze Final IIIA/IIIB (autour de -950) et celui d'Azay-leRideau, avec près de 500 objets ou fragments, puis ceux d'Onzain et de Choussy (plus de 100 objets ou fragments), mais aussi Saint-Genouph, Esvres-sur-Indre, Chédigny, Sublaines ou Fresnes. Plus tard, en Berry, le dépôt de Neuvy-sur-Barangeon comptera pour sa part plus de 600 objets et fragments.

17 Une distinction serait sans doute alors à faire entre les caches de possédants ou de marchands (objets, lingots), les dépôts d'artisans fondeurs (la même chose plus des rebuts divers) ou les dépôts à caractère magico-religieux et votifs (équipements individuels ou sexués). Les dépôts de bronze s'insèrent alors dans des circuits divers de production, d'accumulation et de redistribution. Ils réunissent des pièces regroupées selon diverses circonstances, situation banale ou période de troubles. Ils ont été rassemblés peu après la fabrication des objets, ou plus tard lors de leur échange ou de leur consommation. Leur nature ou leur valeur peut être attachée aux notions d'épargne et de thésaurisation, d'usage et de fonctionnalité, d'abandon ou de perte ou encore de don, de libéralité et d'offrande. Ce sont bien sûr des signes et des marqueurs sociaux possédant une certaine valeur. Et, par "valeur", il faut entendre valeur numéraire ou symbolique, ces notions étant étroitement mêlées aux actes des populations protohistoriques. Tous les dépôts d'objets ne sont donc pas de même nature et ils n'ont pas la même signification. L'avis de G. Cordier aurait été ici très pertinent.

Ces dépôts illustrent en tout cas l'équipement, peut-être idéalisé, des élites sociales ligériennes : richesse des outils, variété des armes, quantité et originalité des parures, exotisme d'objets rares ou curiosités. L'accumulation prend alors un caractère ostentatoire, y compris paradoxalement lorsqu'elle reste enfouie au regard des hommes, comme si le temps avant l'enfouissement était plus important socialement que l'abandon de la richesse des objets. 
19 Genouph, bracelets de type Vaudrevanges (Azay-le-Rideau), bracelets de types Homburg et Balingen (Esvres-sur-Indre), parures annulaires de type Vénat, "abondantes dans les dépôts mais curieusement rares hors dépôts ", pendeloques diverses - dont celle à motifs ornithomorphes de Civray (Cher), multiples épingles à têtes ornementées souvent massives et décorées, sans oublier des bijoux de verre et d'ambre, etc. Comme les découvertes isolées ${ }^{9}$, ces dépôts montrent la place particulière des armes (épée du dépôt d'Orléans, couteaux de type helvétique en Berry et Orléanais) pour cette probable aristocratie dont par ailleurs on sait peu de choses (habitat, hiérarchie, mode de vie, etc.). Cette demande stimule à l'évidence le savoir-faire des artisans (épée à poignée métallique du dépôt de Neuvy-sur-Barangeon « dont il faut chercher l'homologue au Danemark»). L'équipement technique de ses derniers acquiert dans ce contexte une haute qualité y compris symbolique (voir le petit outillage et un coin à estamper à inclusion de fer dans le dépôt de Neuvy-surBarangeon).

Les ateliers régionaux ${ }^{10}$ produisent alors en nombre des pièces plus utilitaires et désormais répandues en assimilant rapidement de nombreux modèles. Ces derniers sont régionaux (hache à douille armoricaine sur le littoral) mais aussi européens (par exemple la hache à ailerons subterminaux dérivant de la hache à ailerons médians « diffusée à partir d'un centre rhodanien jusqu'en Berry et en Orléanais »). Toutes ces productions stimulent aussi les échanges à longue distance et assurent probablement l'introduction de produits exotiques (fibule sicilienne, broche â rôtir et sphéroïde du dépôt de Notre-Dame-d'Or) ou de leurs imitations (racloirs et talons de lance d'affinités launaciennes d'Azay-le-Rideau, haches à appendices latéraux d'inspiration ibérique du dépôt de Mougon, haches italiques du Saumurois et de Choussy, hache d'affinités nordiques de Luynes, faucille à douille de type britannique de Plaimpied-Givaudins, etc.).

21 Les épées sont alors parmi les meilleurs témoins de l'apogée de cette métallurgie et du commerce des armes dans le bassin de la Loire. Ainsi, au Bronze Final III, les types atlantiques - langue de carpe et autres - dominent mais ils s'accompagnent aussi de productions continentales (Rigny-Ussé, Vierzon, Meung-sur-Loire) ou plus rarement italiques (épée à antennes type Tarquinia d'Amboise).

Les artisans du bassin de la Loire sont bien confrontés aux productions étrangères qu'ils attirent, mais elles sont sources de progrès technologiques, de concurrence et d'inspiration. Elles sont alors britanniques (haches de type Gallois, pointes de lance de types britanniques à oeillets, épées de type Ballintober de Rigny-Ussé), mais aussi alpines et helvétiques (Epée de type Monza à Foëcy). Elles confèrent souvent aux dépôts d'objets majoritairement atlantiques des traits continentaux, surtout rhénans (au Bronze Final II, épée de type Nenzingen d'Amboise ; épées Hemigkofen de Chéry, SaintAy, Saint-Martin-d'Auxigny ; épée de type Erlach d'Angers, etc.), voire parfois de « bien plus loin vers l'Est, vers les pays du Danube et jusqu'aux rives de la Baltique » (pièce de harnachement de Chédigny, anneau à figurine vasiforme d'Onzain).

Les objets de bronze révèlent aussi des pratiques sociales novatrices, probablement adoptées par quelques élites et certains pourvoyeurs de richesses. Ainsi la place particulière des cavaleries d'apparat en est une preuve avec de nombreux harnachements en bronze (dépôts de Mirebeau, de Tourtenay, de Chédigny, etc.) et des pièces de chars (dépôt d'Amboise, ou de Mougon avec ses grelots et moyeux de roue, 
sans compter la "luxueuse roue» de Coulon). Ces équipements exceptionnels suggèrent des cérémonies inconnues, peut être sonorisées par de puissants lurs danois (curiosité du dépôt d'Orléans). Et l'apparition de banquets probables est aussi évoquée par le crochet à viande de Coulon, « unique en France à ce jour », voire par les coupes métalliques (Fresnes) et la vaisselle à décors « scandinaves » de Neuvy-sur-Barangeon. On peut alors imaginer le faste des cérémonies pour les funérailles du défunt de Billy dont la célèbre tombe recélait casque, ceinture à pendeloques, feuilles d'or, grains de collier en verre et en ambre. Au-delà des élites, mais partiellement, la société de l'Âge du Bronze final reste toutefois mal connue. Quelques tombes à inhumation modestes (Montgivray, Chédigny, Neuvy-le-Barrois) et des sépultures à incinération (nécropoles de Gièvres, Chissay-en-Touraine), parfois aux céramiques remarquables (assiettescouvercles à décors en guirlandes parfois excisés et surtout figurine ornithomorphe de Tigy) précèdent le «tumulus princier » de Sublaines (avec son urne à décor d'étain et figuration de char) ou celui de Saint-Aoustrille (avec des céramiques à figurations humaines stylisées proches du style du Bourget et que l'on retrouve à Allosnes) ${ }^{11}$.

L'Archéologue du $\mathrm{xxI}^{\mathrm{e}}$ siècle ne saurait être frustré des pans entiers de la société de l'Âge du Bronze qui restent à découvrir dans le bassin de la Loire. La richesse des documents déjà rassemblés doit satisfaire pour l'instant les spécialistes qui possèdent désormais un matériau très avancé pour bâtir une réflexion probablement plus sociologique et environnementale. Et le fort tribut de G. Cordier les y aidera probablement, comme de nombreux inventaires et analyses culturalistes l'ont permis dans bien d'autres régions.

Souhaitons pour lui plaire que les cinquante prochaines années soient encore plus bénéfiques avec des fouilles d'importance d'habitats comme cela a pu être mené en Poitou sous l'impulsion de J.-P. Pautreau et C. Maitay par exemple. Et il est certain que la force de frappe de l'archéologie préventive renouvellera ou complétera comme on le pressent déjà la connaissance des populations de l'Âge du Bronze du bassin de la Loire. Cela serait une élégante façon de remercier l'auteur pour sa contribution dont la lecture est à ce jour à bien des points de vue indispensable.

L'exceptionnelle abondance de figures dues à la plume réputée de Gérard Cordier caractérise d'emblée cet impressionnant ouvrage. La qualité esthétique des dessins va de pair avec une rigoureuse conformité aux détails des objets. Ces figures soutiennent vigoureusement l'exposé de l'implantation et du développement de l'Âge du Bronze en cette vaste région couvrant le bassin de la Loire moyenne et de ses affluents. Elles permettent, visuellement, d'analyser déjà ce qui peut, au sein d'une certaine identité régionale, témoigner des différents passages et influences atlantiques et continentaux au sens large, dans un paysageouvert en toutes directions. L'auteur, délibérément éloigné des vues trop synthétiques vite caduques, s'est attaché d'abord à donner méthodiquement un corpusd'objets et à dégager des constats n'excluant pas certaines lacunes obligées. Il sera impossible désormais de poursuivre quelque étude protohistorique sans référence à ce prodigieux travail.

Venant après plus de 300 publications s'échelonnant sur 65 années, cet imposant ouvrage de 702 pages comprend 458 illstrations dont 380 totalisent les dessins de 
3650 objets ou fragments se répartissant en 1054 haches, 651 bracelets, 567 céramiques, 231 épées, 144 pointes de lance, 83 poignards, 30 faucilles et 890 objets divers. Ce matériel provient des collections de cinquante musées de France et d'Angleterre (le MAN, à Saint-Germain-en-Laye, en a fourni à lui seul 650). 78 cartes explicitées (dont une en couleurs) exposent la distribution géographique. L'inventaire des sites et découvertes concerne 813 communes, avec bien entendu toutes les références utiles. La bibliographie, riche de 1300 titres, s'accompagne de 186 notes infrapaginales. 448 analyses métallographiques (faites au Laboratoire d'Anthropologie préhistorique de la Faculté des Sciences de Rennes), avec renvois aux dessins des objets, donnent les indispensables caractérisations des produits bronziers - locaux ou au contraire éloignés. L'ouvrage s'achève par 22 planches hors-texte réunissant 5 illustrations en couleurs et 53 en noir-et-blanc d'objets et de reproductions de documents anciens. Enfin, une feuille séparée énonce les quelques inévitables errata. Ce monumental ouvrage ne pouvait se concevoir qu'à compte d'auteur afin de pouvoir publier une si abondante iconographie ; de ce fait, le livre est coûteux (mais le « rapport qualité/prix » reste plus que raisonnable). Si La Simarre est bien l'imprimeur, elle n'en est pas l'éditeur comme la couverture le laisse malencontreusement entendre. Aussi le tirage de cet Âge du Bronze est-il minime et il risque d'être vite épuisé. Ce n'est d'ailleurs pas la première fois que G. Cordier a recours à l'auto-édition, par exemple pour l'Inventaire des Mégalithes de la France - Indre-et-Loire dont la réédition n'avait pu être prise en charge par les Editions du CNRS pour Gallia-Préhistoire en 1984, ou bien encore pour Le dépôt de l'Âge du Bronze final du "Petit-Villatte» à Neuvy-sur-Barangeon (Cher) et son contexte régional, en 1996.

La profusion des sources repose sur un patient travail de dépouillement effectué au cours de toute une vie de recherche dans toutes les publications anciennes, pérennes et nouvelles, dans un méticuleux archivage aux multiples entrées. Le don du dessin n'est pas ici la moindre des bonnes dispositions de départ. Depuis l'enfance, Gérard Cordier a toujours pratiqué le dessin à la plume où il a excellé d'emblée, aussi n'est-on pas étonné de retrouver, tout au long des soixante cinq ans passés, des dessins d'un graphisme situé dans l'admirable tradition d'un Adrien de Mortillet dont paraissait, il y a 130 ans, le mémorable Musée préhistorique. Les vues multiples des illustrations de G. Cordier rendent compte plus précisément encore de la technologie de l'objet montré sous toutes ses faces.

Dès sa toute jeunesse, G. Cordier fait preuve d'une insatiable curiosité pour tous vestiges. S'il s'intéresse d'abord aux témoins paléontologiques, la prédilection pour l'univers préhistorique allait vite s'imposer. Il entre en 1942 à la Société préhistorique française (il en est pratiquement le doyen aujourd'hui). Dès l'année suivante, Raymond Vaufrey, alors directeur de la Circonscription archéologique de Paris-Sud (comprenant l'Indre-et-Loire) le pressent pour le Fichier des Mégalithes, tant il est conscient que l'avancée des travaux scientifiques ne peut se faire sans une série d'inventaires spécialisés couvrant l'ensemble des découvertes (fortuites ou non), des produits de fouilles, documents et diverses collections. L'ouvrage, terminé en 1958, inaugure à la fois l'édition des Inventaires des Mégalithes de la France et la collection des Suppléments à Gallia Préhistoire en 1963. Ce travail, alors conduit avec des moyens restreints, devait être refondu et réédité par l'auteur en 1984, après notamment qu'il eût mené les importantes fouilles de Sublaines. 
31 Un autre aspect essentiel de la trajectoire de préhistorien de G. Cordier est son entrée au Cadastre en 1945, où il restera jusqu'en 1966. Pendant vingt ans, ses travaux topographiques seront l'occasion de multipler les découvertes, la publication de ses études s'accompagnant toujours de plans détaillés. Ainsi se confirme la «vertu archéologique » du relevé cadastral comme de l'analyse minutieuse des photographies aériennes, le champ d'action s'étendant du Préacheuléen jusqu'à l'époque galloromaine. Cette ample moisson, loin de décourager notre chercheur par son abondance, va au contraire le stimuler pour approfondir ces domaines, chercher des points de comparaison plus ou moins éloignés - belle enquête géographique - et aussi se préoccuper de la dévolution des séries recueillies. L'action de Gérard Cordier auprès des musées est à cet égard édifiante, exemplaire : son nom figure sur la plaque de marbre des donateurs à l'entrée du château de Saint-Germain-en-Laye, le MAN. Ainsi se trouve bouclé le cycle normal de puis la découverte, l'étude, la publication, la conservation enfin des vestiges archéologiques. Sa vocation de préhistorien est reconnue officiellement par son entrée au CNRS en 1966; elle le confirme dans son activité régionale. Tout naturellement les relations amicales entretenues depuis les années cinquante avec d'autres chercheurs du monde scientifique vont se resserrer; parmi eux, Jacques-Pierre Millotte, Raymond Ricquet et Michel Gruet. Pour ma part, je m'honore de compter parmi les amis de Gérard Cordier depuis le Congrès préhistorique de Poitiers en 1956.

Au moment où Gérard Cordier, dans sa toute jeunesse, s'intéresse définitivement à la préhistoire de la Touraine, son pays natal et de vieille souche, on ne dispose pratiquement que de La Touraine préhistorique de Dubreuil-Chambardel, parue en 1923. Pour que les travaux progressent, il fallait revoir tout l'inventaire bibliographique, reprendre l'étude des séries des musées, des collections privées et des objets épars. C'est à propos de Vernou-sur-Brenne que G. Cordier rencontre R. Riquet en 1952 ; leur étroite collaboration durera trente ans, jusqu'à la mort de ce dernier. La parution de L'Indre-et-Loire préhistorique (sur le modèle du Gard préhistorique de M. Louis), seulement en 1967 après bien des mésintelligences, ouvrait sur un projet plus vaste où seraient rassemblés et complétés les objets donnés par cet ensemble bibliographique de 1967 . On peut dire que dès lors l'inventaire de l'Âge du Bronze de la Touraine et alentours était attendu ; les encouragements ne manquèrent pas, notamment ceux de J.-P. Millote et d'A. Villes qui le réclamèrent dans leurs articles.

Pour réaliser ce colossal travail reposant en majorité sur des objets isolés et des dépôts il fallut consentir à passer des années à de nouvelles enquêtes sur le terrain et dans cinquante musées, consacrer des milliers d'heures aux innombrables dessins, pratiquer un système efficace de recension bibliographique, se livrer à ces nombreuses comparaisons sans lesquelles il n'est pas de conduite valable de la recherche archéologique. L'ouvrage commence par l'exposé des cadres géographique et chronologique, puis par l'historique des études tant en Touraine qu'en Anjou, dans la Sarthe, le Dunois, le Blésois, l'Orléanais mais aussi le Berry, le Poitou. Vient ensuite une précieuse analyse des gîtes métallifères pour la Bretagne, et la Basse-Loire, l'Anjou, le Poitou et la Vendée, le Plateau central. Toutefois, l'exploitation avérée des gîtes d'étain et d'or concerne surtout le pourtour de la zone étudiée.

Ensuite, l'ouvrage se déroule chronologiquement: Campaniforme, Bronze ancien, Bronze moyen, Bronze final. Pour les trois derniers ensembles, sont traités dans l'ordre les dépôts, les objets isolés, les sites funéraires, les habitats. C'est là que se déploie la 
plus abondante, minutieuse et complète documentation participant si remarquablement à la richesse du volume. Y sont inclus des ensembles déjà publiés afin de réunir une documentation jusque là éparse et parfois difficilement accessible. Puis l'inventaire des sites et découvertes offre un irremplaçable appel bibliographique pour les départements d'Indre-et-Loire, Loiret, Loir-et-Cher, Maine-et-Loire, Sarthe et enfin Vienne.

Le résumé regroupe les états de question concernant les périodes considérées dans leur espace géographique. Le Campaniforme se diffuse à partir du littoral vendéen par le nord du Poitou, le Saumurois et le sillon de la Loire. La production vendéenne des haches plates marque le Bronze ancien et leur diffusion vers l'est s'accompagne d'une évolution typologique, et peut-être aussi bien d'un certain retard chronologique. En revanche, quelques objets berrichons paraissent issus de la zone helvétique ou du bassin rhodanien. Deux exceptionnelles sépultures du Loir-et-Cher marquent cette période : Naveil, en relation avec la culture de Remedello (Lombardie) et Saint-Rimay, apparentée aux coffres armoricains. Le Bronze moyen voit s'étendre la production de la hache à talon, avec concentration en Anjou et plus fortement en Saumurois. Une influence vendéenne est sensible pour les haches à forts rebords. Bien entendu, la Loire joue un rôle majeur dans la diffusion de ces produits bronziers. Emergent des formes exceptionnelles et rares comme la hache «bohémienne » de Châteauneuf-sur-Loire ou la hache de parade d'Azay-le-Rideau (en couleurs sur la couverture), peut-être bavaroise ou du Rhin moyen, ou bien encore le « cône d'or » d'Avanton. Les dépôts de la fin du Bronze moyen surprennent par leur richesse, en particulier celui des Ponts-deCé en Anjou, si remarquable par la diversité des décors de ses bracelets. La céramique semble se partager entre influences armoricaine et Kerbschnitt. C'est sans conteste au Bronze final que se rattachent un grand nombre de dépôts et d'objets isolés où se mêlent influences atlantiques et continentales: alpines, rhénanes, helvétiques, danubiennes et jusqu'aux rives de la Baltique avec les éléments de lurs danois d'Orléans, uniques en France. En Saumurois, c'est l'influence normande qui est sensible ; en Berry, la dominante est rhénane et helvétique. Souvent, la mixité des dépôts est avérée. Les objets isolés témoignent d'encore plus sensible échanges lointains. La fig. 456 est un superbe exemple de ce que peut donner une représentation typologique et géographique bien conduite des épées du Bronze final au long de la Loire, où les types atlantiques - langues de carpe et autres - dominent mais où se mêlent des formes continentales ou plus rarement italiques. Quelques tombes plates, des sépultures et nécropoles à incinération ainsi que des tumulus composent les sites funéraires du Bronze final. Leur mobilier céramique abondant et varié se retrouve aussi généreux dans les habitats : chacun peut admirer à Saint-Germain-en-Laye - où l'auteur l'a déposée - l'exceptionnelle urne à décor d'étain et figuration de char de Sublaines. Bien des examens de laboratoire restent à entreprendre tant pour les matériels métalliques que pour les céramiques, et ce malgré que des trouvailles anciennes soient muettes sur leurs conditions d'enfouissement.

Enfin viennent les 1300 références bibliographiques régionales et extra-régionales, fruits d'un laborieux dépouillement (une vie !), les analyses métallographiques, l'index des sites et découvertes répertoriés, le tout s'achevant par des planches hors-texte dont les plus émouvantes sont bien celles où l'on contemple les photos des grands aînés. L'Âge du Bronze dans les pays de la Loire moyenne est là pour témoigner que l'art du dessin n'est pas mort en matière archéologique. Il convient de poser la question de l'archivage et de laconservation de toutes ces précieuses iconographies anciennes ou récentes: le 
support papier reste durable quand le CD demeure électroniquement fragile. On peut regretter que l'auteur n'ait pas été chargé par l'Université d'un enseignement de cette pratique graphique, mais il est vrai aussi qu'un tel cours concernant les outils lithiques préhistoriques a existé durant vingt ans, mais qu'il n'a pas survécu à son fondateur. Puisse la très remarquable publication de cet Âge du Bronze susciter des émules nombreux.

\section{NOTES}

1. G. Cordier a toujours poursuivi la triple ambition de la connaissance, de la conservation et de la divulgation du patrimoine. Son action auprès des services de la Culture, et tout particulièrement des musées, est à ce titre exemplaire.

2. Les travaux les plus récents de l'archéologie préventive ne sont pas pris en compte dans cette somme. Ils n'ont pas fait l'objet de publications pour la plupart. Ainsi les diverses fouilles récentes de l'archéologie préventive ont réuni un très abondant mobilier céramique par le biais d'ensembles issus de dépotoirs volumineux pour la période du Bronze Ancien, du Bronze final III à Villebarou (Loir-et-Cher) et Sublaines (Indre-et-Loire).

3. En 1944 « après plus d'un an de chantier de bûcheronnage dans la Forêt d'Amboise " il inventoriait un site au sud de Saint-Règle et étudiait un puits d'extraction de silex. Et il prônait dès 1950 la lecture des photographies aériennes pour le repérage des sites et des enceintes.

4. On se reportera au récent catalogue d'exposition du Musée Sainte-Croix de Poitiers pour une bibliographie complète: Feux de Camp, un site de hauteur: le Camp Allaric du Néolithique à l'Âge du Fer, 2008, 48 p.

5. Au Grand Ormeau, à Sublaines (Indre-et-Loire), Des restes de constructions de l'Âge du Bronze moyen et des fosses de l'Âge du Bronze final ont livré une importante quantité de tessons de céramique. Pour cette dernière phase on connait un grand bâtiment à abside et à deux nefs, d'une longueur de 12,50 m pour une largeur de 4,50 m. De petites agglomérations à fossés et palissades (Pannes et Vienne-en-Val dans le Loiret, Villebarou dans le Loir-et-Cher), ou de plus grands ensembles organisés autour de bâtiments de ferme, sont connus comme à Port-Sec Sud (Bourges, Cher). En 2007 une superficie de 2,5 ha, située près du marais de Grée et de la Loire a été étudiée à Ancenis, en Loire-Atlantique. Des maisons à plancher et cloisons y sont de plan rectangulaire. Elles mesurent de 15 à $20 \mathrm{~m}$ de long sur 6 à $7 \mathrm{~m}$ de large; elles ont des absides sur leurs pignons occidentaux et une entrée orientée à l'est.

6. L'Archéologie préventive, avec la construction de $101 \mathrm{~km}$ d'autoroute reliant Artenay à Courtenay (A 19), dans le Loiret, a donné lieu à une importante opération en 2006 sur 1380 hectares de tracé. À l'issue des diagnostics et des 30 fouilles prescrites, 2 sites de l'Âge du Bronze (Le Haut de l'Aunette et La Pièce du Mail) ont été fouillés (nécropoles 
de Courcelles à tumulus). Ce seraient des ensembles funéraires familiaux ou lignagers dont le modèle se généraliserait au cours du XIII ${ }^{e}$ siècle avant notre ère.

7. N'a-t-on pas parlé d'influences britanniques pour celle des hallebardes dans la vallée de la Loire, mais en y insérant un épicentre ibérique ayant absorbé des apports continentaux?

8. Cela n'indique pas que les pratiques cultuelles originelles liées à cet objet énigmatique soient nécessairement introduites.

9. B. Poissonnier, en étudiant les découvertes de la Basse Loire, a mis en évidence l'importance des dépôts au sein des pratiques funéraires fluviatiles, évoquant l'ultime " combat mythique » qui nécessitait des armes particulières pour franchir la frontière d'un au-delà figuré par le fleuve.

10. On ne connaît pas d'ateliers métallurgiques dans cette région à l'exception de celui d'Allonnes découvert à l'entrée d'une habitation et probablement domestique.

11. Il faut noter la mise au jour récente d'enclos circulaires de type classique, comme à Pannes (Loiret), ou sous forme d'enclos palissadés, comme à Mignières (Eure-et-Loir), semblables à ceux connus dans l'Est et l'île-de-France. Ils sont parfois associés à des incinérations (par exemple à Montierchaume dans l'Indre) et s'échelonnent sur le Bronze final et le premier âge du Fer. 